has a close relation to the famous integral due to LoMmEL,

$$
\int z C_{\nu}{ }^{2}(z) d z=\left[\frac{z^{2}}{2}\left\{C_{\nu}{ }^{2}(z)-C_{\nu-1}(z) C_{\nu+1}(z)\right\}\right] .
$$

Introducing each of the three kinds of Bessel functions, $J_{v}(z), Y_{v}(z), H_{v}^{(1)}(z)$ and $H_{\nu}{ }^{(2)}(z)$, into the equation (1) in place of $C_{\nu}(z)$, we obtain corresponding kinds of modified quotients, $\mathfrak{Y}_{\nu}(z), \mathfrak{Y}_{\nu}(z), \mathfrak{W}_{\nu}{ }^{(1)}(z)$ and $\mathfrak{S}_{\nu}{ }^{(2)}(z)$, respectively. In various boundary value problems of mathematical physics, we encounter quite often the Bessel functions in quotient forms [2]. It is obvious that the modified quotients defined here give a convenient approach to mathematical analysis and numerical estimates of these problems. Moreover there is a remarkable parallelism between the modified quotients and the trigonometric cotangent and tangent, as there is between the Bessel functions and the sine and cosine. Therefore the modified quotients should have the same raison d'être in cylinder functions as the cotangent and tangent in trigonometric functions.

From the above consideration it seems highly desirable to give permanent symbols to these modified quotients, to collect their formulae and to construct their tables. To this end an attempt was made [3], but further cooperation and criticism of interested workers are necessary.

MORIO ONOE

Institute of Industrial Science

University of Tokyo

Chiba City, Japan

1. G. N. Watson, Theory of Bessel Functions, Cambridge Univ. Press, 1922, p. 82 ff.

2. For example:

DENNISON BANCROFT, "The velocity of longitudinal waves in cylindrical bars," Phys. Rev., 59,1941, p. 588 .

G. E. Hudson, "Dispersion of elastic waves in solid circular cylinders," Phys. Rev., 63, 1943, p. 46.

H. SUHL \& L. R. WALKER, "Topics in guided-wave propagation through gyromagnetic media," Bell System Tech. J., 33, 1954, p. 579.

3. M. ONOE, "Formulae and tables, modified quotients of cylinder functions," Report of the Institute of Industrial Science, University of Tokyo, No. 32, 1955.

\title{
Flip-flop as Generator of Random Binary Digits
}

The aim of the present note is to show that a well known electronic element of digital computers, the flip-flop, may be used for generating a series of random binary digits with equal probabilities.

Let us consider a flip-flop as shown on fig. 1 and let A and B denote two possible stable states of the flip-flop. If we switch on the contact $S$, the flip-flop will be randomly set in one of its states $\mathrm{A}$ or $\mathrm{B}$. We may obtain by the aid of the flip-flop a sequence of $2 k$ random elements $X_{1}, X_{2}, \cdots, X_{2 k}$, (abbreviated $\left\{X_{2 k}\right\}$ ), where

$$
X_{j}=\left\{\begin{array}{l}
\mathrm{A}, \text { if } j \text {-th switching on the contact } S \text {, set flip-flop in state A } \\
\mathrm{B}, \text { if } j \text {-th switching on the contact } S \text {, set flip-flop in state B }
\end{array}\right.
$$

and $1 \leq j \leq 2 k$. 


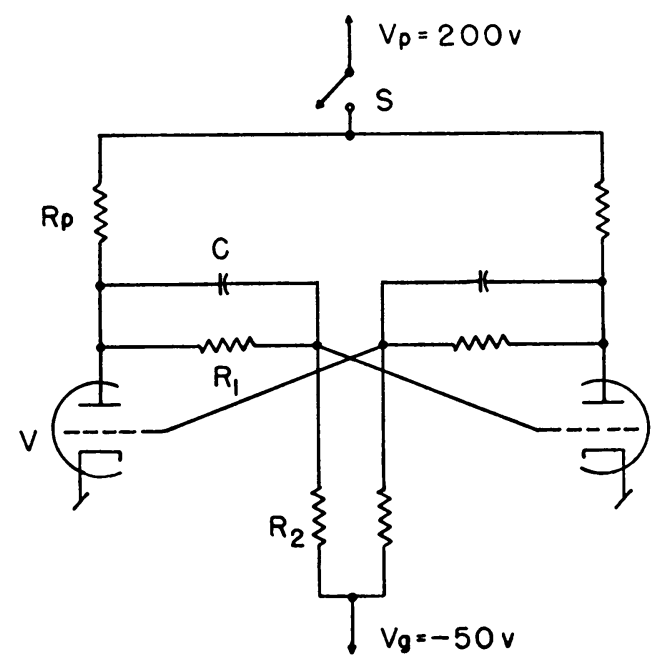

$$
\begin{aligned}
& R_{p}=10 \mathrm{k} \Omega \\
& R_{1}=160 \mathrm{k} \Omega \\
& R_{2}=50 \mathrm{k} \Omega \\
& C=50 \mu \mu \mathrm{fd} \\
& V=1 / 26 \mathrm{SN} 7
\end{aligned}
$$

Fig. 1.

In this way we may obtain a finite random series of A and B which are statistically independent. One series produced by the aid of a flip-flop is given below:

\section{AABAABBABBABBBABBAABABABBABAABABABBAA \\ BABABBBBBBBBBABBBABAABBB.}

Let $\left\{Y_{k}\right\}$ be the sequence of $k$ pairs of elements of $\left\{X_{2 k}\right\}$ such that $Y_{i}=X_{2 i-1}, X_{2 i}$, where $1 \leq i \leq k$. Omitting in $\left\{Y_{k}\right\}$ all elements of the form $\mathrm{AA}$ and $\mathrm{BB}$ we obtain a third sequence whose elements are the pairs AB and BA only, denoted in the following by 0 and 1 respectively.

Let $p_{j}(\mathrm{~A})$ and $p_{j}(\mathrm{~B})$ denote probabilities that $j$-th switching on of contact $S$ set flip-flop in state $A$ or $B$ respectively and suppose that $p_{j}(\mathrm{~A})$ and $p_{j}(\mathrm{~B})$ are asymmetric, say $p_{j}(\mathrm{~A})>p_{j}(\mathrm{~B})$. Supposing that the flip-flop does not change its properties during two successive switchings, we may write

$$
\begin{aligned}
& p_{2 i-1}(\mathrm{~A})=p_{2 i}(\mathrm{~A}) \\
& p_{2 i-1}(\mathrm{~B})=p_{2 i}(\mathrm{~B}) .
\end{aligned}
$$

From 1 and 2 we have

$$
p_{2 i-1}(\mathrm{~A}) \cdot p_{2 i}(\mathrm{~B})=p_{2 i-1}(\mathrm{~B}) \cdot p_{2 i}(\mathrm{~A}) .
$$

\section{Because}

$$
p_{2 i-1}(\mathrm{~A}) \cdot p_{2 i}(\mathrm{~B})=p_{i}(0)
$$


and

$$
p_{2 i-1}(\mathrm{~B}) \cdot p_{2 i}(\mathrm{~A})=p_{i}(1)
$$

therefore

$$
p_{i}(0)=p_{i}(1)
$$

where $p_{i}(0)$ and $p_{i}(1)$ are probabilities of zeros and ones in the $i$-th place of the sequence $\left\{Y_{k}\right\}$.

The procedure above described may be used for production of binary random numbers by automatic digital computers. In this case the manual switch $S$ must be replaced by an electronic switch, of course.

Warszawa, Poland

Z. PAWLAK

\section{Numerical Solution of the Schroedinger Equation for Central Fields}

A fast program has been written for the ILLIAC to integrate the radial SCHROEDINGER equation

$$
\begin{gathered}
u^{\prime \prime}(r)-\left[l(l+1) / r^{2}+2 V(r)-E\right] u=0 \\
\text { boundary conditions: } u(0)=0 \quad u(r) \text { bounded }
\end{gathered}
$$

for any well-behaved potential $V(r)$. More generally the program can integrate any linear second-order differential equation which can be put in the form

$$
u^{\prime \prime}(r)-g(r) u(r)=q(r)
$$

with $q(r)$ vanishing at zero and infinity, $r^{2} g(r)$ bounded at zero, and $g(r)$ bounded for large $r$ [1].

A distinctive feature of the program is the use of the Noumerov [2], [3] method for the integration. This is faster than techniques (such as the RunGEKUTTA) which depend on making an estimate of one or more forward points and improving this by an iteration scheme. Here there is no iteration, but the error in the "estimate" of each forward point is of eighth order in the step-size $h$, so that the truncation error may still be kept small. The essence of the method is the elimination of all odd powers of $h$ from the TAYLOR expansion about any point by working with three points instead of two, followed by a change of dependent variable which removes the $h^{4}$-term. The calculation of a forward point to order $h^{8}$ thus requires the value of the dependent variable at the six preceding points.

For an equation in the form (2), the required new dependent variable is

$$
y=u-\left(h^{2} / 12\right)(g u+q) .
$$

The prescription for calculating forward points is

$$
y_{n+1}=2 y_{n}-y_{n-1}+h^{2} \frac{g_{n} y_{n}+q_{n}}{1-\frac{1}{12} h^{2} g_{n}}-\frac{1}{240} \delta^{6} y_{n},
$$

